\title{
Trichoderma harzianum Improves Defense Against Fusarium oxysporum by Regulating ROS and RNS Metabolism, Redox Balance, and Energy Flow in Cucumber Roots
}

\author{
Shuang-Chen Chen, ${ }^{1,2, \dagger}$ Jing-Jing Ren, ${ }^{1}$ Hong-Jiao Zhao, ${ }^{1}$ Xiang-Li Wang, ${ }^{1}$ Tai-Hang Wang,,${ }^{1,2}$ Sun-Da Jin, ${ }^{1}$ \\ Zhong-Hong Wang, ${ }^{1,2}$ Chong-yang Li,${ }^{1}$ Ai-Rong Liu, ${ }^{1}$ Xiao-Min Lin, ${ }^{1}$ and Golam Jalal Ahammed ${ }^{1, \dagger}$ \\ ${ }^{1}$ College of Forestry, Henan University of Science and Technology, Luoyang 471023, People's Republic of China \\ 2 Department of Plant Science, Agricultural and Animal Husbandry College, Tibet University, Linzhi 860000, People's Republic of China \\ Accepted for publication 30 January 2019.
}

\begin{abstract}
Plant survival in the terrestrial ecosystem is influenced by both beneficial and harmful microbes. Trichoderma spp. are a group of filamentous fungi that promote plant growth and resistance to harmful microbes. Previously, we showed that the genus Trichoderma could effectively suppress Fusarium wilt in cucumber. However, the mechanisms that underlie the effects of the genus Trichoderma on plant defense have not been fully substantiated. Two essential metabolic pathways, such as the ascorbate (AsA)-glutathione (GSH) cycle and the oxidative pentose phosphate pathway (OPPP), have been shown to participate in plant tolerance to biotic stressors; nevertheless, the involvement of these pathways in Trichoderma-induced enhanced defense remains elusive. Here, we show that Trichoderma harzianum could alleviate oxidative and nitrostative stress by minimizing reactive oxygen species (ROS; hydrogen peroxide and superoxide) and reactive nitrogen species (nitric oxide [NO]) accumulation, respectively, under Fusarium oxysporum infection in

cucumber roots. The genus Trichoderma enhanced antioxidant potential to counterbalance the overproduced ROS and attenuated the transcript and activity of NO synthase and nitrate reductase. The genus Trichoderma also stimulated $S$-nitrosylated glutathione reductase activity and reduced $S$-nitrosothiol and $S$-nitrosylated glutathione content. Furthermore, the genus Trichoderma enhanced AsA and GSH concentrations and activated their biosynthetic enzymes, $\gamma$-GCS and L-galactono-1,4-lactone dehydrogenase. Interestingly, the genus Trichoderma alleviated Fusarium-inhibited activity of glucose-6-phosphate dehydrogenase and 6-phosphogluconate dehydrogenase, enzymes involved in the OPPP. Such positive regulation of the key enzymes indicates the adequate maintenance of the AsA-GSH pathway and the OPPP, which potentially contributed to improve redox balance, energy flow, and defense response. Our study advances the current knowledge of Trichoderma-induced enhanced defense against $F$. oxysporum in cucumber.
\end{abstract}

Plant survival in the terrestrial ecosystem is impacted by both biotic and abiotic factors. Among multiple biotic factors, one group of microbial organisms negatively affects normal growth and existence of plants, commonly termed harmful microbes, whereas the other group facilitates plant growth, development, and adaptation to stress, referred to as beneficial microbes (LópezRáez et al. 2017). Trichoderma spp. are a group of filamentous saprophytic fungi that promote plant growth and resistance to various disease causal agents, including plant-pathogenic fungi (Chen et al. 2016; Gupta et al. 2014; Howell 2003; Singh et al. 2014). Trichoderma spp. are ubiquitous in the soil around the world. Many strains of the genus Trichoderma are used as effective biocontrol agents for disease management in greenhouse as well as field crop production, and thus, they are considered as an

${ }^{\dagger}$ Corresponding authors: S.-C. Chen; chen_shuangchen@126.com, and G. J. Ahammed; ahammed@haust.edu.cn

Funding: This project was funded by National Key Research and Development Program of China grant 2018YFD1000800; National Natural Science Foundation of China grants 31471867, 31872092, and 31872157; Henan Natural Science Foundation grants 182300410046 and 182300410090 ; International Cooperation Project of Henan Provincial Science and Technology Department grant 172102410050; Henan University of Science and Technology Research Start-Up Fund for New Faculty grant 13480058; and the Key Laboratory of Horticultural Crop Growth and Quality Control in Protected Environment of Luoyang City.

*The $\boldsymbol{e}$-Xtra logo stands for "electronic extra" and indicates that four supplementary figures are published online.

The author(s) declare no conflict of interest.

C 2019 The American Phytopathological Society opportunistic fungal group (Gupta et al. 2014; Lorito et al. 2010; Singh et al. 2014, 2018). Evidence is being accumulated regarding the mechanisms of Trichoderma-induced enhanced tolerance to plant pathogens. Trichoderma spp. can suppress pathogens through competition for space and nutrients (Howell 2003; Singh et al. 2014). Many of them show antibiotic activity that can kill the pathogens. Trichoderma spp.-secreted lytic enzymes can degrade cell walls of the pathogens. They can also induce systemic resistance to pathogens. However, several lines of evidence suggest that mycoparasitism and antibiosis are not the main mechanism of biocontrol in many cases (Lorito et al. 2010). This implies that the underlying defensive mechanisms of the plants induced by the genus Trichoderma are far from being fully substantiated.

The genus Fusarium comprises a large group of plant-pathogenic filamentous ascomycete fungi that cause wilt, blight, rot, and canker diseases of many plant species in both agricultural and natural ecosystems (Ma et al. 2013). In particular, Fusarium oxysporum includes a number of strains that cause vascular wilt diseases in $>100$ economically important crops all over the world (Gordon 2017; Ma et al. 2013). Some strains of F. oxysporum are able to penetrate the host root asymptomatically; afterward, they colonize vascular tissue and cause massive wilting, necrosis, and chlorosis of aerial plant parts (Gordon 2017). Reactive oxygen species (ROS) accumulation increases at the site of Fusarium infection, and the expression patterns of many transcription factor genes during colonization seem to be similar to those of oxidative stress (van der Does and Rep 2017). ROS production is a universal response of plant cells that mediates the perception of microbe-associated molecular patterns (Gupta et al. 2014; Ma et al. 2013). Moreover, the virulence of some plant-pathogenic fungi depends on the 
ability to respond to ROS. Inoculation with certain strains of $F$. oxysporum increases the levels of hydrogen peroxide $\left(\mathrm{H}_{2} \mathrm{O}_{2}\right)$ and lipid peroxidation in tomato (Zehra et al. 2017). A comparative proteomics study on cucumber root after infection with $F$. oxysporum f. sp. cucumerium revealed the involvement of jasmonic acid as well as redox signaling modules in plant resistance to $F$. oxysporum (Zhang et al. 2016a, b).

Nitric oxide (NO) is a fascinating signal molecule, playing diverse roles in a plethora of metabolic and physiological processes (Cánovas et al. 2016; Lindermayr 2018). In plants, NO mediates multiple aspects relating to plant growth and stress responses (Gupta et al. 2014; Lv et al. 2017; Zhang et al. 2019). Moreover, higher organisms often use NO to defend against microbial pathogens, such as fungi and bacteria (Brown et al. 2009; Cánovas et al. 2016). In contrast, NO may also appear as a harmful molecule to plants because of its ability to react with proteins, which causes nitrosylation and nitrosative stress (Gupta et al. 2014; Lv et al. 2017). Plant-pathogenic fungi also use NO as a signaling molecule to infect plants. Therefore, nitrostative stress used by plants may be either beneficial or harmful to plant or pathogen depending on the specific plant-pathogen interaction (Brown et al. 2009; Cánovas et al. 2016). For instance, Arabidopsis mutants that lack the flavohemoglobin GLB1 show increased NO levels and enhanced defense against Botrytis cinerea infections (Mur et al. 2012). However, decreased NO accumulation increases the susceptibility of tobacco (Nicotiana tabacum) to the genus Colletotrichum (Asai et al. 2008). Rapid and increased NO production in roots is essential for the necrotrophic disease development after inoculation with F. oxysporum in the genus Arabidopsis (Gupta et al. 2014). Thus, in parallel to ROS generation, NO is rapidly generated in plants after host-incompatible as well as -compatible pathogen attacks (Brown et al. 2009; Cánovas et al. 2016). In addition to ROS, reactive nitrogen species (RNS) affect plant growth and stress responses by modulating the signaling networks either independently or synergistically (Chen et al. 2017; Lindermayr 2018; Lv et al. 2017).
The oxidative pentose phosphate (OPP) pathway plays a crucial role in multiple biological processes in plants by generating nicotinamide adenine dinucleotide phosphate (NADPH) required for carbon fixation, nitrogen assimilation, and redox poise (Hu et al. 2019; Kruger and von Schaewen 2003). Notably, NADPH is also essential for the biosynthesis of nucleotides, amino acids, and fats as well as maintenance of an ample intracellular glutathione (GSH) pool, which functions to balance cellular ROS in plants (Chen et al. 2017; Kuźniak et al. 2017). The glucose-6-phosphate (G6P) is converted to 6-phosphogluconolactone (6PG) by the catalysis of the glucose-6-phosphate dehydrogenase (G6PDH), the chief NADPH-generating enzyme ( $\mathrm{Hu}$ et al. 2019). The 6PG is enzymatically converted to ribulose-5-phosphate by the action of a second enzyme, namely 6-phosphogluconolactone dehydrogenase (6PGDH), through oxidative decarboxylation. G6PDH as well as 6PGDH function to generate the NADPH, and then, the pentose phosphate pathway (PPP) is accomplished through a nonoxidative phase (Ramos-Martinez 2017). In Arabidopsis, loss of cytosolic G6PDH attenuates plant resistance to root knot nematodes, whereas G6PDH-dependent NADPH production and subsequent ROS signaling mediate plant basal defense against root knot nematodes (Hu et al. 2019).

Cucumber (Cucumis sativus L.) is an important vegetable, having high economic value worldwide. Nonetheless, cucumber production is greatly hampered by a deadly fungal disease called Fusarium wilt disease that often appears as an epidemic (Estifanos Tsegaye et al. 2018). Among different Fusarium spp. that cause Fusarium wilt in cucumber, F. oxysporum is considered as the major causal agent owing to its severity, virulence, and widespread occurrence (Chen et al. 2016; Estifanos Tsegaye et al. 2018). Considering the safety of the environment and human health, biocontrol methods have been introduced to control Fusarium wilt, especially in organic agriculture. Previously, we showed that certain strains of the genus Trichoderma could effectively suppress Fusarium wilt in cucumber (Chen et al. 2016). However, the
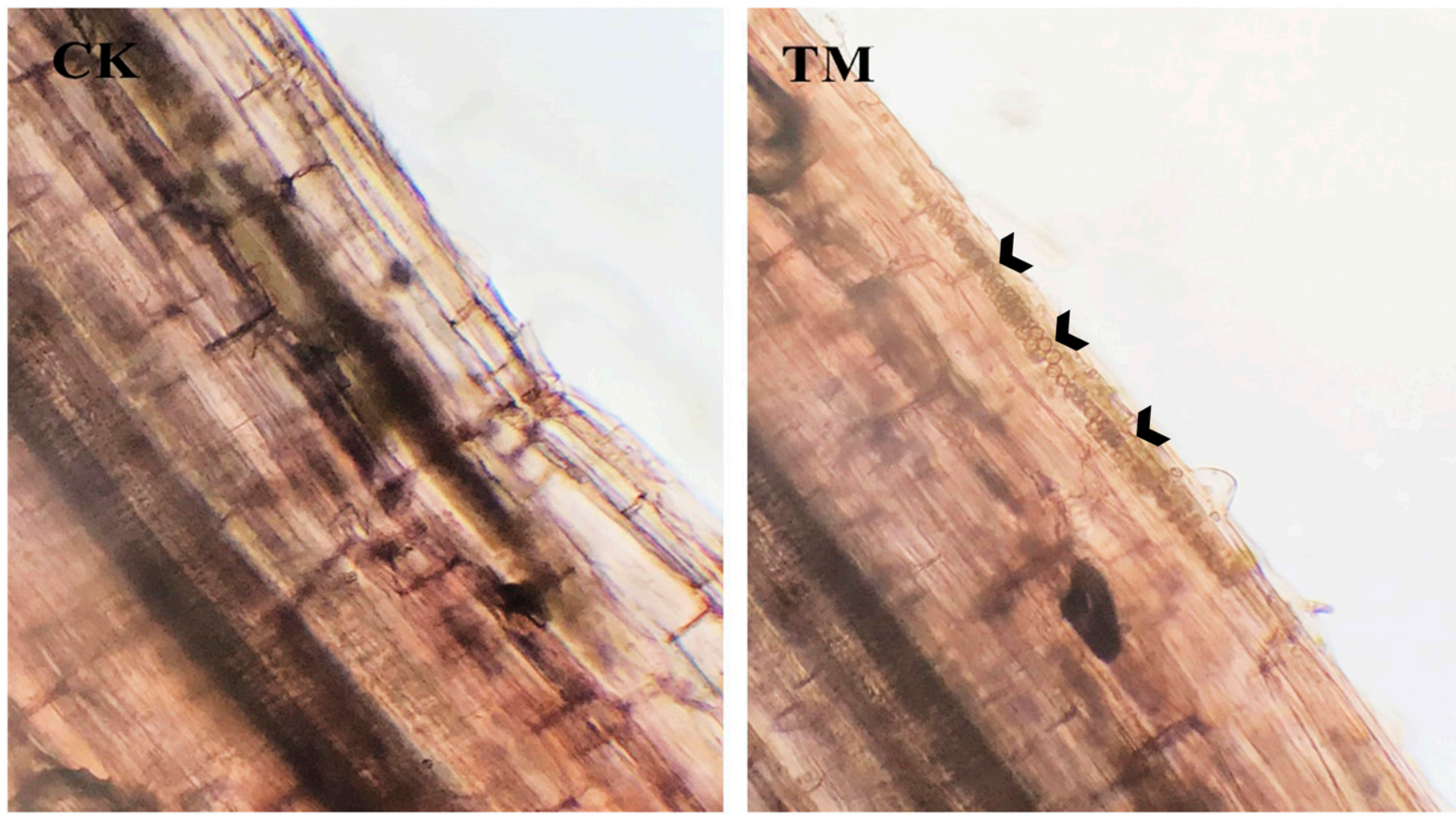

Fig. 1. Penetration and colonization of Trichoderma harzianum within the root of cucumber by the smaller hyphae. Black arrowheads show the localization of T. harzianum within the root of cucumber. CK, mock control; TM, inoculation with only T. harzianum. 
mechanisms that underlie the Trichoderma-induced enhanced tolerance to the genus Fusarium still remain largely unknown. Here, we examine the effect of Trichoderma-Fusarium interaction on ROS and RNS metabolism, antioxidant defense, the ascorbate
(AsA)-GSH cycle, and the key enzymes of the OPP pathway in cucumber. Our results show a role for Trichoderma harzianum in reducing $F$. oxysporum-induced ROS and RNS, which potentially alleviated oxidative and nitrostative stress, respectively, in
A

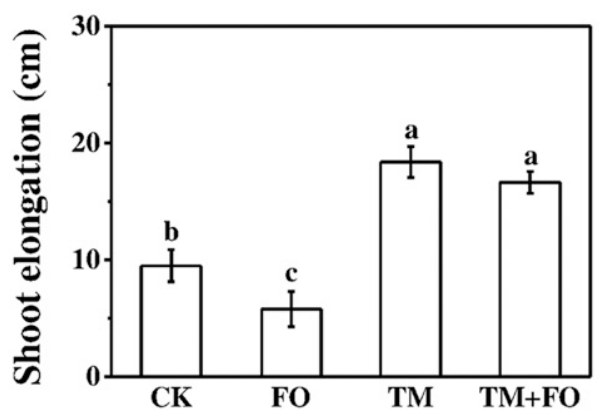

B

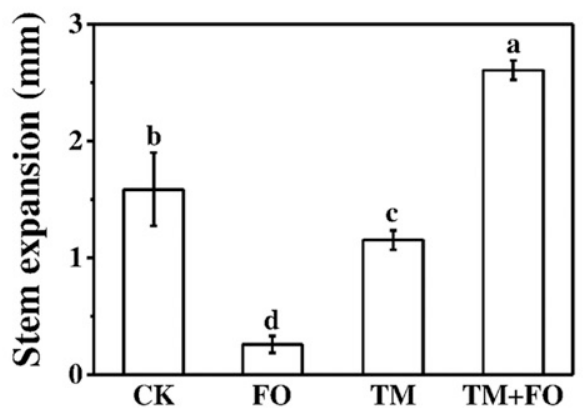

C

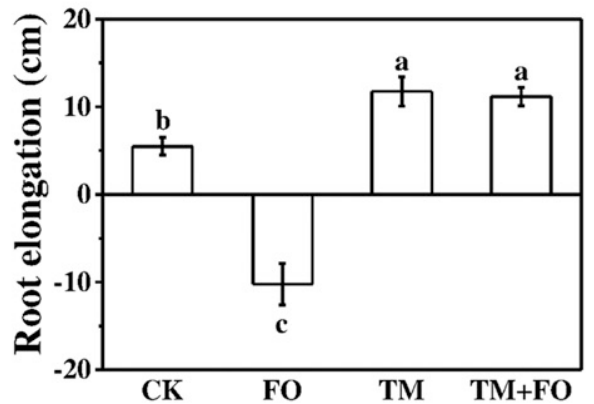

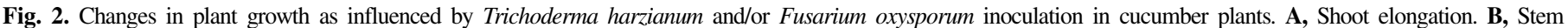

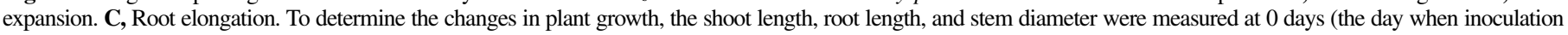

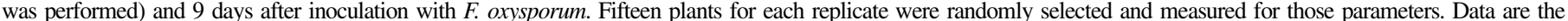

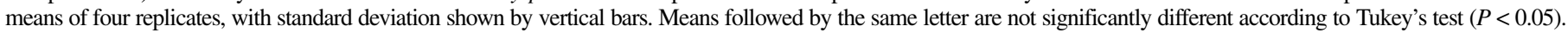
CK, mock control; FO, inoculation with only F. oxysporum; TM, inoculation with only T. harzianum; TM + FO, coinoculation with T. harzianum and F. oxysporum.
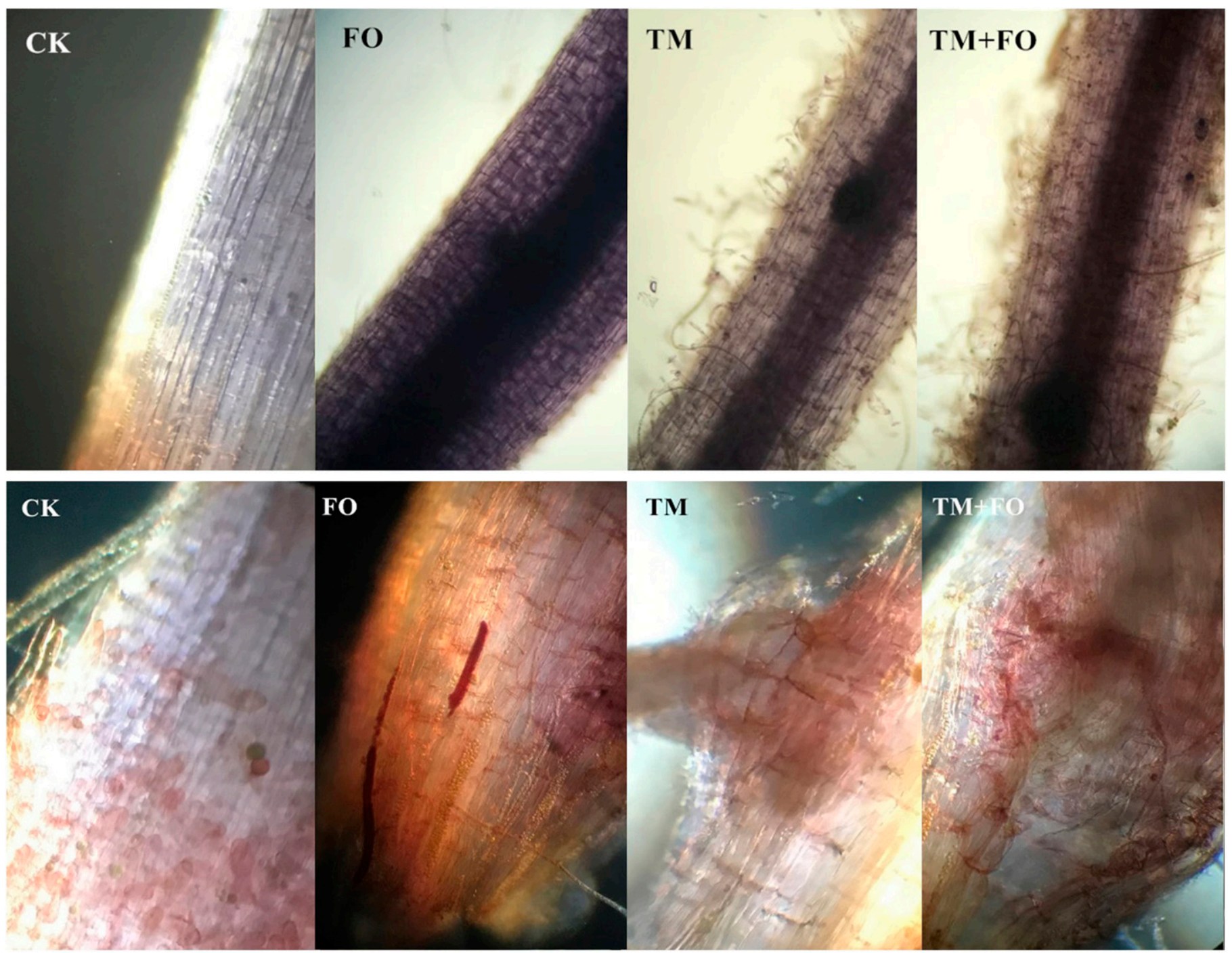

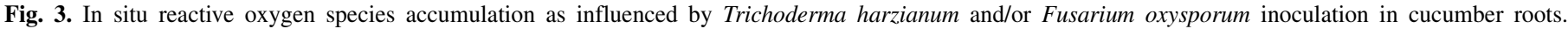

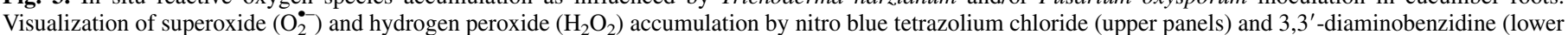

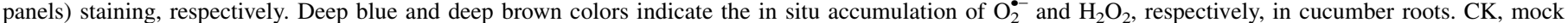
control; FO, inoculation with only F. oxysporum; TM, inoculation with only T. harzianum; TM + FO, coinoculation with T. harzianum and F. oxysporum. 
cucumber roots. Moreover, T. harzianum attenuated F. oxysporuminduced inhibition in the AsA-GSH cycle and the OPP pathway and improved antioxidant potential of plants. This study advances the current knowledge of Trichoderma-induced enhanced defense against $F$. oxysporum in plants.

\section{MATERIALS AND METHODS}

Plant species, fungal strains, and experimental treatments. Cucumber (cultivar Jinyan No. 4) was used as plant material in this study. This cucumber genotype is susceptible to $F$. oxysporum. The culture of collected $F$. oxysporum f. sp. cucumerinum isolates was accomplished on a Fusarium-selective medium of Komada (1975) and confirmed on the basis of conidial morphology at the microscopic level (Booth 1971). T. harzianum strain (TH58) was collected from $\mathrm{Xu}$ Tong (Institute of Biotechnology, Zhejiang University, Hangzhou, China).

After surface sterilization of cucumber seeds with sodium hypochlorite solution $(1 \% \mathrm{wt} / \mathrm{vol})$, the seeds were sown in perlite for germination. Sixty seedlings per treatment were cultured in a hydroponics batch containing half-strength Enshi nutrient solution (10 liters solution per plastic tank). The experiment was carried out in a greenhouse under $28^{\circ} \mathrm{C}$ (day) and $20^{\circ} \mathrm{C}$ (night) temperature regimes, a relative humidity of $85 \%$, and a 12 -h light period (600 $\mu \mathrm{mol} \mathrm{m}{ }^{-2} \mathrm{~s}^{-1}$ photosynthetic photon flux density [PPFD]). When the seedlings were at the four-leaf stage, inoculation of one-half of the seedlings was done with $T$. harzianum through the addition of conidia (T. harzianum TH58) into the culture solution. The final concentration of conidia in culture solution was $10^{8}$ conidia per milliliter. After 3 days of TH58 inoculation, conidial suspension of $F$. oxysporum was added into the nutrient solution of one-half of $T$. harzianuminoculated seedlings and one-half of mock-inoculated seedlings. The final concentration of conidia (the genus Fusarium) in culture solution was $10^{6}$ conidia per milliliter. The details of the preparation of $F$. oxysporum suspension and treatment were described previously (Chen et al. 2016). To determine the changes in plant growth, the shoot length, root length, and stem diameter were measured at 0 days (the day when inoculation was performed) and 9 days after inoculation with $F$. oxysporum. Successful colonization of the genus Trichoderma in cucumber roots was confirmed by microscopic observation (Fig. 1). It should be noted that, at 9 days postinoculation (dpi), cucumber plants infected with $F$. oxysporum showed obvious disease symptoms, such as leaf yellowing and wilting symptoms, and then, the experiment was terminated. The following formulas were used for the calculation of the incidence of disease: disease incidence (percentage $)=$ number of infected plants/number of total plants $\times 100$, the percent disease index $(\mathrm{PDI})=\left(\sum\right.$ (rating score $\times$ number of plants rated)/total number of plants $\times$ highest rating score $) \times 100$, and the control effect (percentage $)=[($ control PDI - treatment PDI $) /$ control PDI] $\times 100$ (Chen et al. 2016).

In situ ROS accumulation and biochemical quantification of ROS and malondialdehyde levels. In situ $\mathrm{H}_{2} \mathrm{O}_{2}$ and superoxide $\left(\mathrm{O}_{2}^{--}\right)$accumulation was visualized by histochemical staining of cucumber roots with 3,3'-diaminobenzidine (DAB) and nitro blue tetrazolium chloride (NBT), respectively. Briefly, freshly harvested roots were rapidly immersed in a DAB solution $(1 \mathrm{mg} / \mathrm{ml}$, $\mathrm{pH} 3.8$ ) followed by incubation at $25^{\circ} \mathrm{C}$ for $6 \mathrm{~h}$. The stained roots were subsequently submerged in ethanol $(96 \%)$ and boiled for 10 min to clear pigments (Ahammed et al. 2018). The reaction of DAB and $\mathrm{H}_{2} \mathrm{O}_{2}$ produced a deep brown polymerization product. In situ $\mathrm{O}_{2}^{--}$accumulation in roots was visualized by staining the root sections with NBT solution $(0.5 \mathrm{mM}, \mathrm{pH}$ 6.0) in the dark (Liszkay et al. 2004). $\mathrm{H}_{2} \mathrm{O}_{2}$ concentration in roots was measured using a peroxidase (POD)-based spectrophotometric assay following the method of Willekens et al. (1997). The $\mathrm{O}_{2}^{\bullet-}$ production rate was measured according to Elstner and Heupel (1976). To measure lipid peroxidation in roots, the malondialdehyde (MDA) equivalents were quantified based on the thiobarbituric acid reaction (Landi
2017). Relative electrolyte leakage (percentage) in root was determined by measuring the electric conductivity of detached roots before and after autoclaving as described by Ahammed et al. (2019).

Determination of NO, $S$-nitrosothiol, and $S$-nitrosylated GSH content and activity of $S$-nitrosylated GSH reductase, NO synthase, and nitrate reductase. NO content was analyzed based on the protocol of hemoglobin assay according to Pasqualini et al. (2009), whereas total $S$-nitrosothiol (SNO) content was measured following the method of Saville (1958). The concentration of $S$-nitrosylated glutathione (GSNO) was quantified following the method of Airaki et al. (2012) as described previously (Chen et al. 2017). The activity of $S$-nitrosylated glutathione reductase (GSNOR) was analyzed as per Sakamoto et al. (2002) as described previously (Lv et al. 2017). Briefly, for the extraction of GSNOR, $50 \mathrm{mM}$ HEPES buffer ( $\mathrm{pH}$ 8.0) containing 20\% glycerol, $10 \mathrm{mM}$ magnesium chloride, $1 \mathrm{mM}$ ethylenediaminetetraacetic acid, $1 \mathrm{mM}$ benzamidine, $1 \mathrm{mM}$ egtazic acid, and $1 \mathrm{mM}$ aminocaproic acid was used. After centrifugation of the homogenates at $4^{\circ} \mathrm{C}$ at $16,000 \times g$ for $15 \mathrm{~min}$, the resulting supernatants were desalted using a spin column. Each reaction system constituted the following

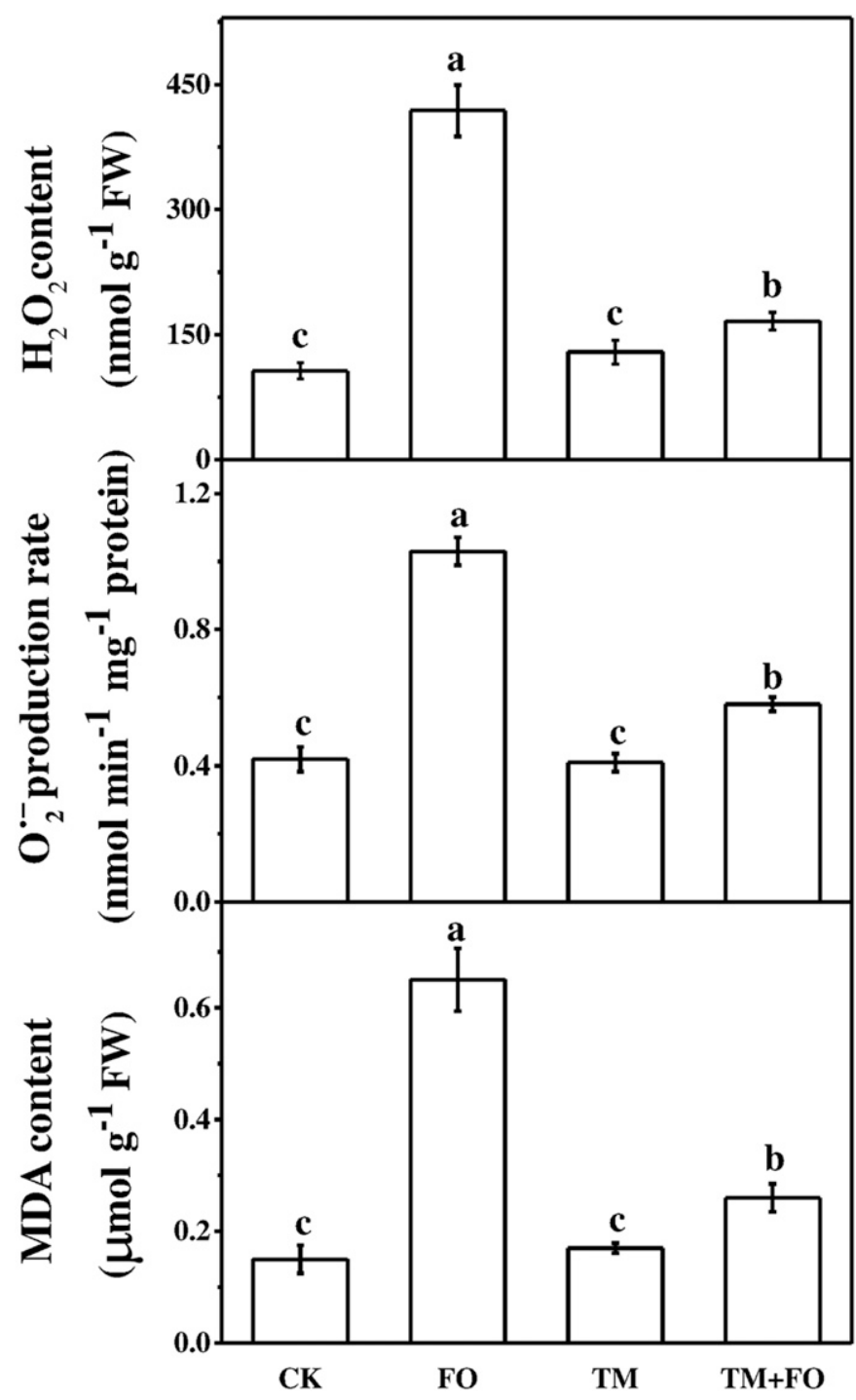

Fig. 4. Quantitative measurements of hydrogen peroxide $\left(\mathrm{H}_{2} \mathrm{O}_{2}\right)$, superoxide $\left(\mathrm{O}_{2}^{\circ-}\right)$, and malondialdehyde (MDA) levels. Data are the means of four replicates, with standard deviation shown by vertical bars. Means followed by the same letter are not significantly different according to Tukey's test $(P<0.05)$. FW, fresh weight; CK, mock control; FO, inoculation with only Fusarium oxysporum; TM, inoculation with only Trichoderma harzianum; TM + FO, coinoculation with $T$. harzianum and $F$. oxysporum. 
components: protein samples, reaction buffer $(20 \mathrm{mM}$ Tris- $\mathrm{HCl}$ containing $2 \mathrm{mM}$ reduced nicotinamide adenine dinucleotide [NADH] and $0.5 \mathrm{mM}$ ethylenediaminetetraacetic acid), and $400 \mathrm{mM}$ GSNO. GSNOR activity was analyzed by recording the decay of NADH at $340 \mathrm{~nm}$. Nitric oxide synthase (NOS) activity was analyzed with an NOS assay kit, and the optical density (OD) was monitored at $540 \mathrm{~nm}$ (Chen et al. 2017). The method of Foyer et al. (1998) was used to analyze the activity of nitrate reductase (NR) as previously described by Lv et al. (2017).

Determination of AsA-GSH cycle components (enzymes and antioxidants). We assayed the activity of glutathione reductase (GR; EC 1.6.4.2) based on the decline in the absorbance of reduced NADPH at $340 \mathrm{~nm}$ following the method of Foyer and Halliwell (1976). The activity of monodehydroascorbate reductase (MDHAR; EC 1.6.5.4) was analyzed by monitoring the oxidation rate of NADH at $340 \mathrm{~nm}$ (Rao and Ormrod 1995). The activity of other antioxidant enzymes was assayed as described previously (Ahammed et al. 2018). GSH was extracted from cucumber roots, and the concentrations of the reduced form of GSH and the oxidized form of glutathione (GSSG) were assayed following the method of Rao and Ormrod (1995). The concentration of GSSG was subtracted from the concentration of total GSH to obtain the GSH concentration. AsA concentration was measured according to Law et al. (1983). The concentration of reduced AsA was subtracted from the concentration of total AsA (AsA + DHA) to obtain DHA concentration. The activity of L-galactono-1,4-lactone dehydrogenase (GaILDH) was assayed following the method described by Tabata et al. (2001). The activity of $\gamma$-GCS was analyzed according to Shan and Liang (2010).

Estimation of the activity of enzymes involved in PPP. The activity of G6PDH was assayed using a spectrophotometric method that relied on the reduction of NADPH (Hu et al. 2019). The activity of 6PGDH was measured by monitoring the reduction of NADPH at $340 \mathrm{~nm}$ (Valderrama et al. 2006). The activity of
NADP-ICDH was assayed on the basis of reduction of NADPH. The reaction system for the analysis of NADP-dependent malic enzyme (NADP-ME) activity consisted of $50 \mathrm{mM}$ Tris $\mathrm{HCl}(\mathrm{pH}$ 7.5), $0.5 \mathrm{mM}$ NADPH, $10 \mathrm{mM}$ malate, $10 \mathrm{mM}$ magnesium chloride, and $10 \mu$ l enzyme extract (Badia et al. 2015).

RNA extraction and quantitative reverse transcriptionpolymerase chain reaction for gene expression analysis. Root samples for the quantitative reverse transcription-polymerase chain reaction (qRT-PCR) assay were harvested at 1 dpi with $F$. oxysporum. Trizol reagent was used for the extraction of total RNA from root tissues according to the instructions of the manufacturer. We used DNase treatment (BBI) to remove genomic DNA. The ReverTra Ace qPCR RT Kit was used to reverse transcribe total RNA $(1 \mu \mathrm{g})$ following the manufacturer's instruction. qRT-PCR was carried out on an iCycler iQ real-time PCR detection system (Bio-Rad). The reaction mixture $(25 \mu \mathrm{l})$ constituted SYBR Green PCR Master Mix $(12.5 \mu \mathrm{l})$, diluted complementary DNA $(1 \mu \mathrm{l})$, and $0.2 \mu \mathrm{M}$ primers (forward and reserve), and the following polymerase chain reaction conditions were maintained: denaturation at $95^{\circ} \mathrm{C}$ for $3 \mathrm{~min}$ and 40 cycles of denaturation at $95^{\circ} \mathrm{C}$ for $10 \mathrm{~s}$ and annealing at $58^{\circ} \mathrm{C}$ for $45 \mathrm{~s}$. The primers used for amplification were designed based on the expressed sequence tag (EST) sequences: NOS (encoding NOS, XM_004142199.2), 5'CGGTGGGAGATTGGTGTCTA-3' and 5'-GGCACATTCCCTG ATTTCCC-3'; NR3 (encoding NR, JQ692875.1), 5'-ATGGTGGAA GTGAAAGGC-3' and 5'-ACAACAAACATCTCCGTCT-3'; GR (encoding GR, EF530128.1), 5'-GTCATCACCCAAAGCAG-3' and 5'-ATAGTTTCCCATCCACATT-3'; MDHAR (encoding MDHAR, Cucsa.240950), 5'-ATGATCTACCCTGAACCG-3' and 5'-CC TCCGACACCCACAAC-3'; actin, 5'-TGGACTCTGGTGATG GTGTTA-3' and 5'-CAATGAGGGATGGCTGGAAAA-3' . Transcript levels were normalized to actin, and relative gene expression was calculated according to Livak and Schmittgen (2001).

Statistical analysis. Data represent mean values of four replicates of each treatment, and the experiment itself was repeated

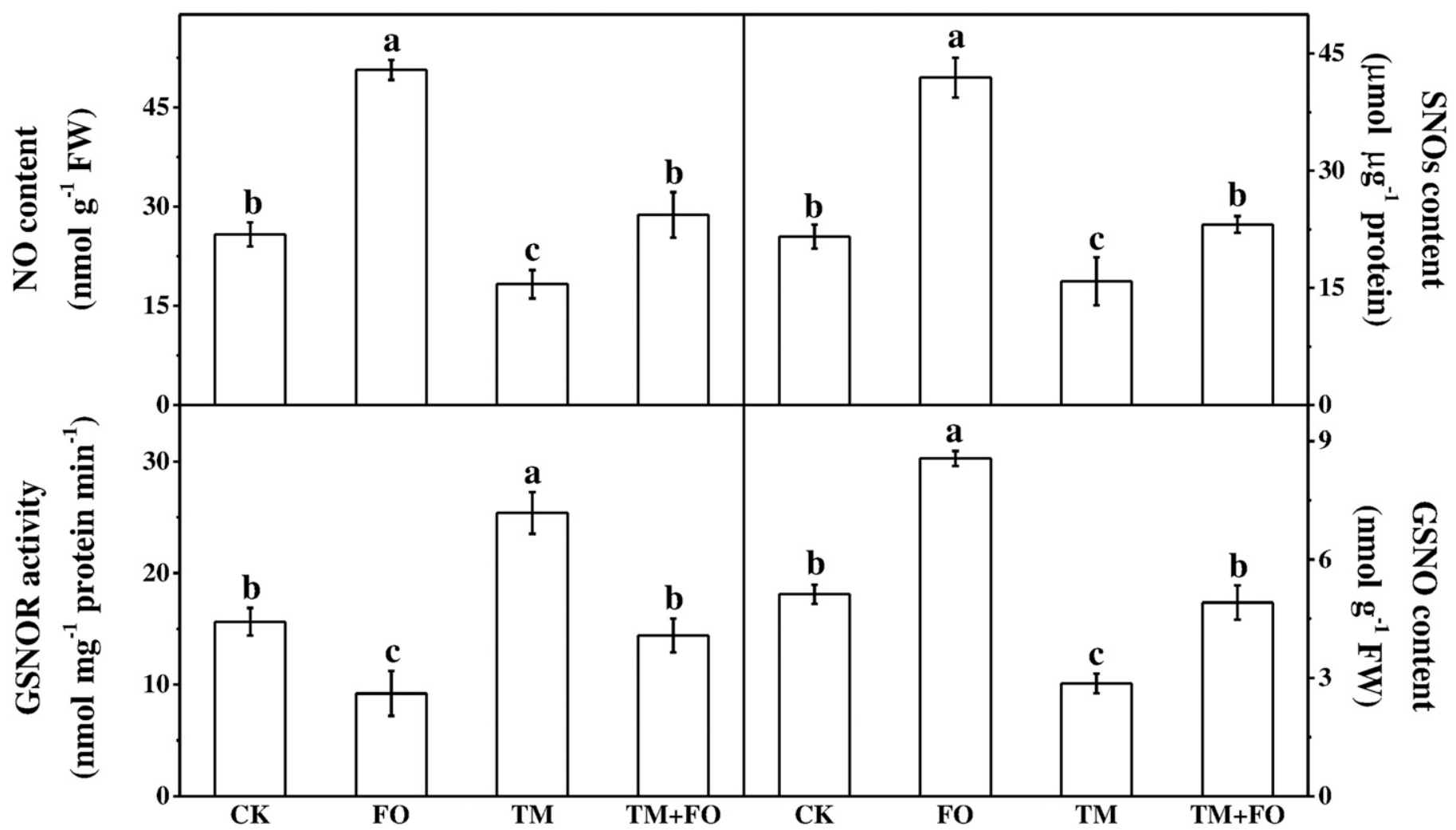

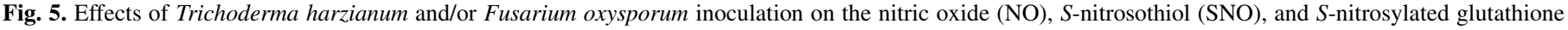

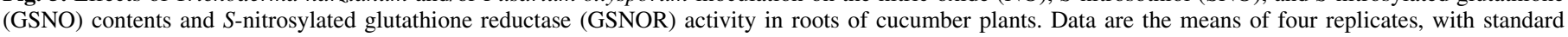

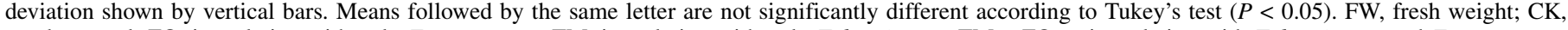

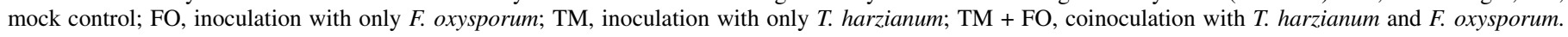


three times independently. Data were subject to analysis of variance, and the values were expressed as mean \pm standard deviation. Means were compared using a Tukey's test at $P \leq 0.05$.

\section{RESULTS}

T. harzianum alleviates $F$. oxysporum-induced inhibition of elongation growth. To assess the effect of $T$. harzianum and/or $F$. oxysporum on plant growth, we first measured the changes in shoot length, root length, and stem diameter after treatment with T. harzianum and/or F. oxysporum. As shown in Figure 2A, shoot elongation was significantly inhibited by $F$. oxysporum infection. Accordingly, F. oxysporum-inoculated cucumber plants showed the wilting phenotype, which is a characteristic feature of Fusarium wilt disease (Supplementary Fig. S1). Notably, before the evaluation of biometric parameters of plant growth promotion, we measured incidence of disease and found that $F$. oxysporum inoculation infected $96.7 \%$ of plants, whereas $T$. harzianum $+F$. oxysporum inoculation infected only $30.0 \%$ of plants. Moreover, the control effect (percentage) of $T$. harzianum $+F$. oxysporum was significantly higher than that of $F$. oxysporum alone (Supplementary Fig. S2). Notably, T. harzianum remarkably stimulated shoot elongation in $F$. oxysporum-inoculated cucumber plants. Shoot elongation in $T$. harzianum $+F$. oxysporum-inoculated plants was 2.9-fold greater than that of only $F$. oxysporum-inoculated cucumber. Meanwhile, $F$. oxysporum inoculation resulted in a negative root elongation, implying that root length became shorter than that of the control, possibly because of rotting or decomposition (Fig. 2C). In contrast, T. harzianum treatment alone or with $F$. oxysporum significantly increased root elongation compared with that of control or F. oxysporum alone, respectively. Unlike the effect of $T$. harzianum alone on shoot and root elongation, T. harzianum alone slightly decreased stem diameter expansion (Fig. 2B). Similarly, F. oxysporum-only treatment also caused a 6.1-fold decrease in stem diameter expansion compared with the control. However, the treatment with T. harzianum $+F$. oxysporum resulted in a 10 -fold increase in stem diameter expansion compared with that of $F$. oxysporum alone treatment.

Excessive ROS accumulation retarded cucumber growth under Fusarium wilt. To explore the mechanism of $F$. oxysporuminduced root growth inhibition, we next visualized in situ $\mathrm{O}_{2}^{\circ-}$ and $\mathrm{H}_{2} \mathrm{O}_{2}$ accumulation in roots by NBT and DAB staining, respectively. As shown in Figure 3, upper panels, F. oxysporum inoculation caused an intensive accumulation of $\mathrm{O}_{2}^{\circ-}$ as reflected by the deep blue color deposits. Consistently, DAB staining showed that $F$. oxysporum inoculation increased $\mathrm{H}_{2} \mathrm{O}_{2}$ accumulation as evidenced by the deep brown color in roots (Fig. 3, lower panels). However, T. harzianum treatment with $F$. oxysporum remarkably attenuated $F$. oxysporum-induced ROS accumulation in roots. Biochemical analysis of ROS levels showed that $F$. oxysporum inoculation resulted in 3.9- and 2.5-fold increases in $\mathrm{H}_{2} \mathrm{O}_{2}$ and $\mathrm{O}_{2}^{\circ-}$ concentrations, respectively, in cucumber roots. Consistent with the histochemical observation, T. harzianum treatment with F. oxysporum significantly decreased ROS levels, because $\mathrm{H}_{2} \mathrm{O}_{2}$ and $\mathrm{O}_{2}^{--}$contents in $F$. oxysporum-inoculated roots were 2.5- and 1.8 -fold greater than that in $T$. harzianum $+F$. oxysporum, respectively (Fig. 4, top and middle panels). To examine the membrane damage caused by excessive ROS, we measured MDA concentration and electrolyte leakage (percentage) in roots (Fig. 4, bottom panel and Supplementary Fig. S3). The results showed that F. oxysporum inoculation resulted in 4.3- and 3.4-fold increases in root MDA content and electrolyte leakage, respectively, compared with that of control. However, T. harzianum treatment drastically decreased MDA content and electrolyte leakage in F. oxysporuminoculated cucumber roots, which was consistent with the T. harzianum-induced reduction in ROS accumulation in roots (Figs. 3 and 4).
NO metabolism is involved in Trichoderma-enhanced resistance to $\boldsymbol{F}$. oxysporum in tomato roots. Plant-pathogen interactions have been shown to be mediated by RNS (Brown et al. 2009; Cánovas et al. 2016). Therefore, we measured NO, SNO, and GSNO content in cucumber roots (Fig. 5). The results showed that F. oxysporum inoculation resulted in 2.0-, 1.9-, and 1.7-fold increases in NO, SNO, and GSNO content, respectively. On the contrary, T. harzianum-only treatment significantly reduced their contents, and T. harzianum $+F$. oxysporum inoculation resulted in 1.7-, 1.8-, and 1.7fold decreases in NO, SNO, and GSNO contents, respectively, compared with that of only $F$. oxysporum treatment in cucumber roots. Unlike the content of NO, SNO, and GSNO, the GSNOR activity was significantly decreased by $F$. oxysporum inoculation, whereas T. harzianum-only treatment showed an opposing effect. Notably, T. harzianum treatment with $F$. oxysporum caused a 1.6-fold increase in GSNOR activity compared with that of only $F$. oxysporum treatment.

To confirm the source of $\mathrm{NO}$ induced by $F$. oxysporum, we analyzed the activity of NO-generating enzymes, such as NOS and NR (Lindermayr 2018; Lv et al. 2017). F. oxysporum-only treatment caused a 1.5-fold increase in the activity of NOS and NR, whereas $T$. harzianum-only treatment decreased NOS activity but did not alter NR activity (Fig. 6). Meanwhile, T. harzianum inoculation with $F$. oxysporum significantly attenuated $F$. oxysporuminduced elevation in NOS and NR activity. This implies that NO produced via NOS and NR pathways potentially increased the infection severity of $F$. oxysporum; however, $T$. harzianum minimized $F$. oxysporum-induced NO accumulation by inhibiting NOS and NR activity in cucumber roots (Fig. 6).

Trichoderma-induced enhanced tolerance to $F$. oxysporum is attributed to efficient redox homeostasis. To assess cellular redox state after inoculation with $T$. harzianum and/or $F$. oxysporum, we measured both oxidized and reduced AsA and GSH contents. As shown in Figure 7, F. oxysporum inoculation decreased the reduced

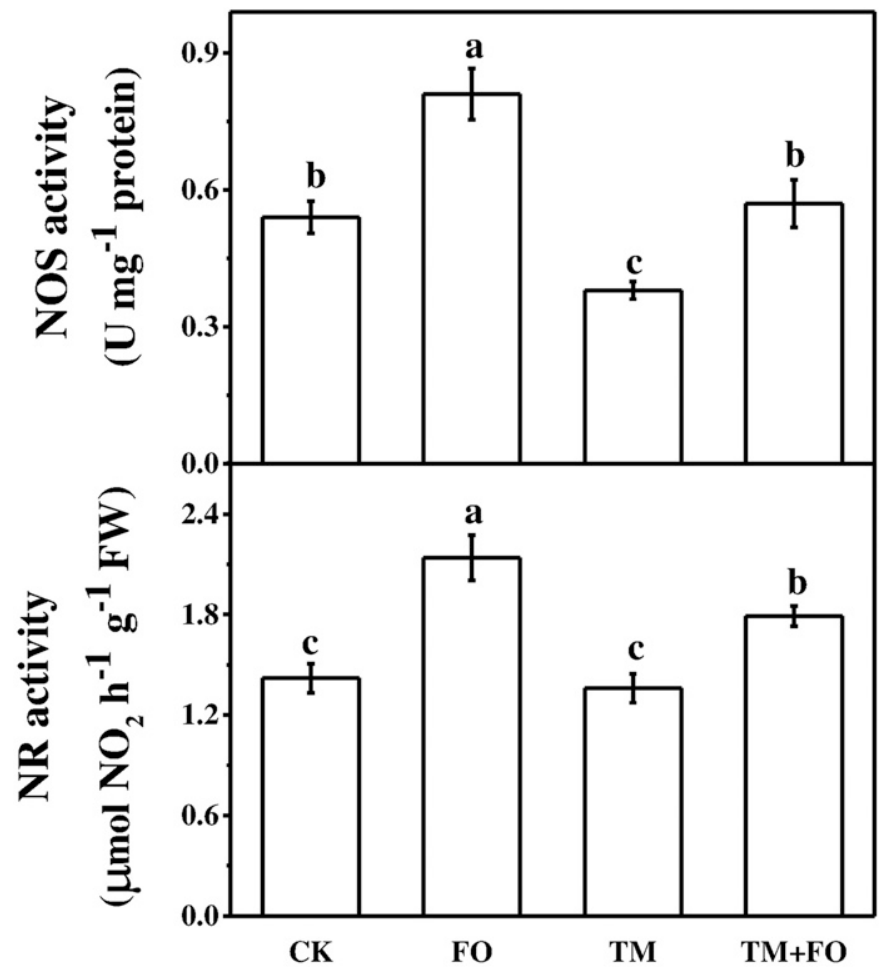

Fig. 6. Changes in the activity of nitric oxide synthases (NOS) and nitrate reductase (NR) as influenced by Trichoderma harzianum and/or Fusarium oxysporum inoculation in cucumber roots. Data are the means of four replicates, with standard deviation shown by vertical bars. Means followed by the same letter are not significantly different according to Tukey's test $(P<0.05)$. FW, fresh weight; CK, mock control; FO, inoculation with only F. oxysporum; TM, inoculation with only $T$. harzianum; $\mathrm{TM}+\mathrm{FO}$, coinoculation with $T$. harzianum and F. oxysporum. 
AsA and GSH contents but induced the oxidized AsA (DHA) and GSH (GSSG) content in cucumber roots. However, T. harzianum alone or $T$. harzianum $+F$. oxysporum treatment significantly increased the concentrations of AsA and GSH compared with those of the control and F. oxysporum-only treatment, respectively. The concentrations of AsA and GSH in T. harzianum + F. oxysporumtreated roots showed 1.8- and 1.3-fold increases, respectively, compared with that of only $F$. oxysporum inoculation. Meanwhile, T. harzianum-only treatment did not alter DHA and GSSG contents, but $T$. harzianum $+F$. oxysporum treatment significantly attenuated

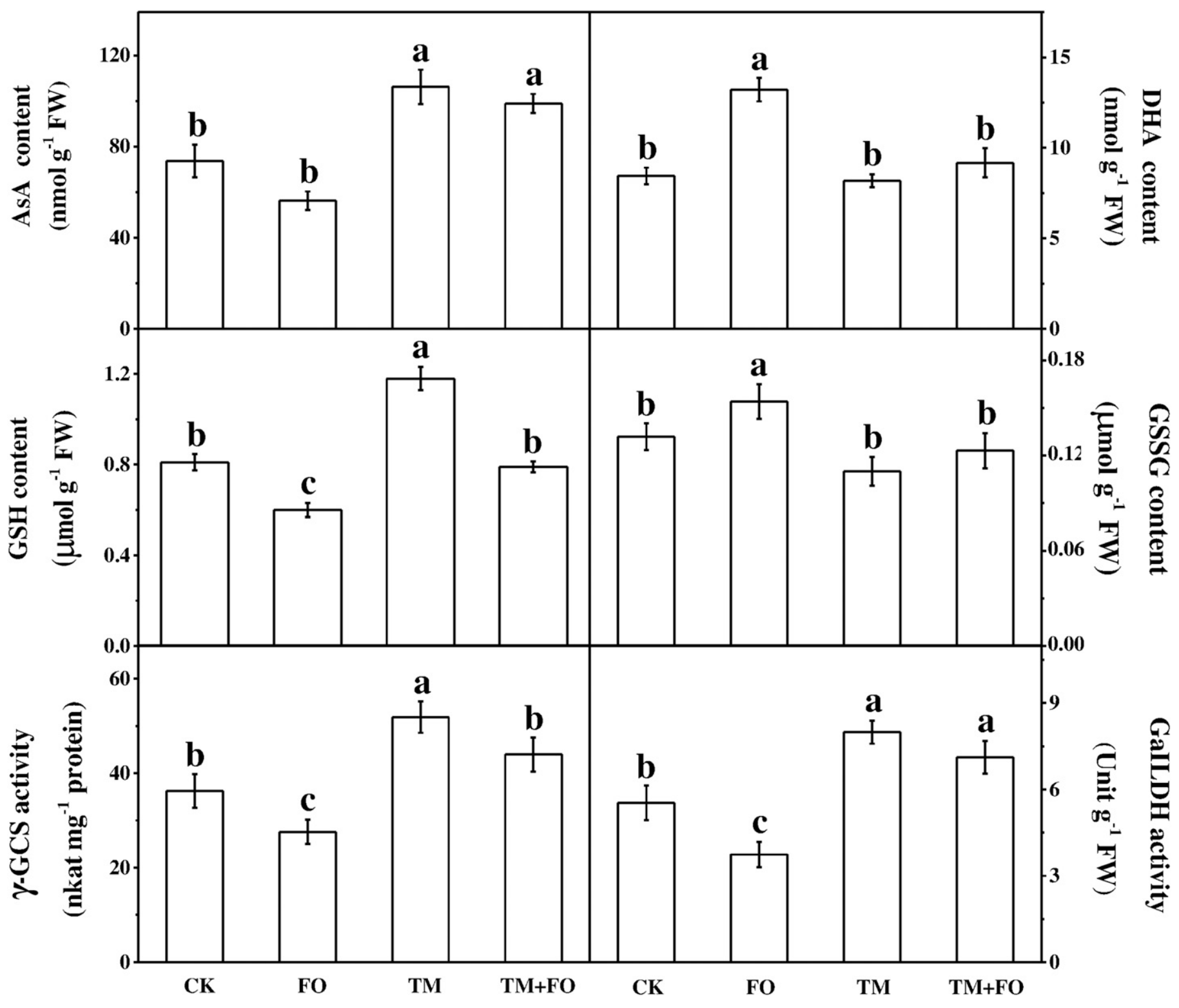

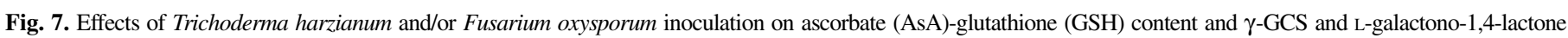

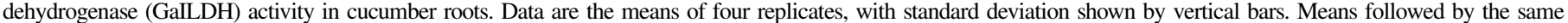

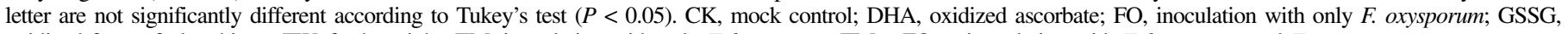
oxidized form of glutathione; FW, fresh weight; TM, inoculation with only T. harzianum; TM + FO, coinoculation with T. harzianum and F. oxysporum.

TABLE 1. Effect of Trichoderma harzianum and/or Fusarium oxysporum inoculation on the activities of enzymes relating to antioxidant system and pentose phosphate pathway in cucumber roots ${ }^{\mathrm{z}}$

\begin{tabular}{|c|c|c|c|c|c|c|}
\hline Treatment & $\begin{array}{c}\text { GR activity } \\
\left(\mu \mathrm{mol} \cdot \mathrm{min}^{-1} \mathrm{mg}^{-1}\right. \\
\text { protein })\end{array}$ & $\begin{array}{l}\text { MDHAR activity } \\
\left(\mu \mathrm{mol} \cdot \mathrm{min}^{-1} \mathrm{mg}^{-1}\right. \\
\text { protein })\end{array}$ & $\begin{array}{l}\text { G6PDH activity } \\
\left(\mu \mathrm{mol} \cdot \mathrm{min}^{-1} \mathrm{mg}^{-1}\right. \\
\text { protein })\end{array}$ & $\begin{array}{c}\text { 6PGDH activity } \\
\left(\mu \mathrm{mol} \cdot \mathrm{min}^{-1} \mathrm{mg}^{-1}\right. \\
\text { protein })\end{array}$ & $\begin{array}{l}\text { NADP-dependent } \\
\text { isocitrate } \\
\text { dehydrogenase activity } \\
\left(\mu \mathrm{mol} \cdot \mathrm{min}^{-1} \mathrm{mg}^{-1}\right. \\
\text { protein })\end{array}$ & $\begin{array}{l}\text { NADP-ME activity } \\
\left(\mu \mathrm{mol} \cdot \mathrm{min}^{-1} \mathrm{mg}^{-1}\right. \\
\text { protein })\end{array}$ \\
\hline CK & $0.075 \pm 0.008 b$ & $0.266 \pm 0.011 \mathrm{c}$ & $34.67 \pm 2.85 b$ & $20.15 \pm 2.46 b$ & $34.25 \pm 1.16 \mathrm{~b}$ & $26.36 \pm 2.18 b$ \\
\hline $\mathrm{FO}$ & $0.052 \pm 0.012 \mathrm{c}$ & $0.159 \pm 0.023 \mathrm{~d}$ & $25.72 \pm 3.68 c$ & $13.68 \pm 1.04 \mathrm{c}$ & $25.28 \pm 3.01 \mathrm{c}$ & $17.47 \pm 0.95 c$ \\
\hline $\mathrm{TM}$ & $0.125 \pm 0.010 \mathrm{a}$ & $0.432 \pm 0.037 \mathrm{a}$ & $44.56 \pm 5.41 b$ & $33.63 \pm 4.79 a$ & $44.82 \pm 5.37 \mathrm{a}$ & $38.52 \pm 4.41 \mathrm{a}$ \\
\hline $\mathrm{TM}+\mathrm{FO}$ & $0.106 \pm 0.016 \mathrm{a}$ & $0.371 \pm 0.025 b$ & $39.15 \pm 2.06 \mathrm{~b}$ & $28.90 \pm 2.73 \mathrm{a}$ & $31.69 \pm 2.29 b$ & $30.97 \pm 2.93 b$ \\
\hline
\end{tabular}

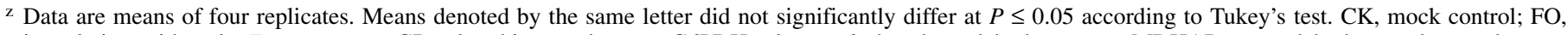
inoculation with only F. oxysporum; GR, glutathione reductase; G6PDH, glucose-6-phosphate dehydrogenase; MDHAR, monodehydroascorbate reductase; NADP-ME, NADP-dependent malic enzyme; 6PGDH, 6-phosphogluconolactone dehydrogenase; TM, inoculation with only T. harzianum; TM + FO, coinoculation with $T$. harzianum and $F$. oxysporum. 
the F. oxysporum-induced elevation in DHA and GSSG contents in cucumber roots. The activity of $\gamma$-GCS and GaILDH was inhibited by F. oxysporum inoculation. However, $T$. harzianum treatment with $F$. oxysporum caused 1.6- and 1.3-fold increases in the activity, respectively, compared with that of only $F$. oxysporum treatment (Fig. 7). Notably, T. harzianum-only treatment also significantly increased the activity of $\gamma$-GCS and GaILDH compared with those of both control and $F$. oxysporum-only treatment.

The genus Trichoderma stimulates activity of enzymes related to antioxidant defense and PPP to enhance defense against $\boldsymbol{F}$. oxysporum. Analysis of antioxidant enzymes, such as GR and MDHAR, showed that $F$. oxysporum treatment resulted in 1.4- and 1.7-fold decreases, respectively, compared with that of control (Table 1). However, T. harzianum inoculation increased the antioxidant enzyme activity in $F$. oxysporum-treated cucumber roots. We also analyzed the activity of superoxide dismutase (SOD), POD, catalase (CAT), and ascorbate peroxidase (APX) in cucumber roots (Supplementary Fig. S4). We found that the activity of SOD, POD, CAT, and APX significantly increased in $T$. harzianum $+F$. oxysporum compared with that in F. oxysporum alone, suggesting that T. harzianum inoculation remarkably improved antioxidant defense in $T$. harzianum $+F$. oxysporum plants. Meanwhile, F. oxysporum caused 1.4- and 1.5-fold decreases in the activity of G6PDH and $6 \mathrm{PGDH}$, respectively, but $T$. harzianum $+F$. oxysporum treatment resulted in 1.5- and 2.1-fold increases in their activity, respectively, compared with that of $F$. oxysporum-only inoculation. We also analyzed the activity of NADP-ICDH and NADP-ME, which was inhibited by $F$. oxysporum but promoted by $T$. harzianum inoculation in cucumber roots (Table 1). All of these results indicate that $T$. harzianum-induced enhanced defense against $F$. oxysporum was attributed to $T$. harzianum-induced stimulation of the antioxidant enzyme activity, the AsA-GSH cycle, and the OPP pathway.

Changes in the expression of ROS and RNS metabolismrelated genes in cucumber roots. To further explore the mechanism of T. harzianum-induced ROS and RNS metabolism, we assayed the expressions of four relevant genes in cucumber roots. The expressions of NOS and NR3 upregulated, whereas those of $G R$ and MDHAR downregulated in roots after inoculation with F. oxysporum. However, T. harzianum inoculation significantly downregulated the expressions of NOS and NR3 and upregulated $G R$ and MDHAR expressions in cucumber roots (Fig. 8). Furthermore, the transcript levels of NOS and NR3 in T. harzianumpreinoculated (but F. oxysporum-infected) seedlings showed 3.1- and 2.2-fold decreases, respectively, whereas those of $G R$ and MDHAR showed 7.77- and 7.9-fold increases, respectively, compared with F. oxysporum-only treatment. Our findings provide convincing evidence that the inoculation with $T$. harzianum could reduce ROS and NO accumulation by modulating the expressions of ROS scavenging- and NO production-related genes, leading to enhanced defense against $F$. oxysporum.

\section{DISCUSSION}

For millennia, Trichoderma spp. have been sustaining agricultural yields naturally through their beneficial effects on crops (Lorito et al. 2010). However, scientifically, their ability to suppress or control disease was first recognized in the early 1930s (Howell 2003). Since then, studies have revealed their enormous potential to boost crop growth, yield, and resistance to multiple stressors (Ahmad et al. 2015; Chen et al. 2016; Lorito et al. 2010). Trichoderma spp. are used as effective biocontrol agents, and a large number of Trichoderma-based biopesticides are available for the management of plant diseases (Bonanomi et al. 2018; López-Ráez et al. 2017; Singh et al. 2014). However, it is becoming increasingly clear that our understanding of the mechanisms of Trichodermainduced biocontrol has been incomplete (Lorito et al. 2010; Singh et al. 2014). In this study, we found that $T$. harzianum could alleviate oxidative and nitrostative stress by improving the metabolism of
ROS and RNS under Fusarium infection in cucumber roots (Figs. 2 to 5). Although the genus Trichoderma enhanced antioxidant potential to efficiently scavenge overproduced ROS under Fusarium wilt, the attenuation of both NR and NOS activities by the genus Trichoderma potentially minimized NO production in cucumber roots (Figs. 5 and 6). These phenomena were closely associated with the Trichoderma-induced adequate maintenance of the AsA-GSH cycle and the OPP pathway through the positive regulation of relevant enzymes activity (Fig. 7 and Table 1).

Trichoderma-induced beneficial effects on plants are largely characterized by remarkable growth promotion, which has greatly increased the attractiveness of these fungi for general use in crop production (Lorito et al. 2010). In this study, the genus Trichoderma alone or in combination with $F$. oxysporum significantly increased growth of shoot, root, and stem compared with that of control or only Fusarium-inoculated cucumber plants, respectively (Fig. 2). Our results are in agreement with the study of Zehra et al. (2017), which showed that different strains of T. harzianum could increase shoot length, root length, and stem diameter in tomato plants. Although the detailed mechanisms of Trichoderma-induced growth promotion have not been fully substantiated, it is believed that the genus Trichoderma can improve nutrient availability and uptake of the plants, which largely contribute to plant growth promotion (Singh et al. 2014). Moreover, Trichoderma-induced improved plant growth could be attributed to enhanced photosynthesis or photosynthetic efficiency (López-Ráez et al. 2017; Lorito et al. 2010).

ROS are continuously produced through aerobic metabolism; however, stress conditions trigger ROS production (Foyer 2018). The general concept of ROS homeostasis is that low levels of ROS participate in cell signaling toward acclimation and improved stress tolerance, whereas excess ROS beyond elimination capacity of

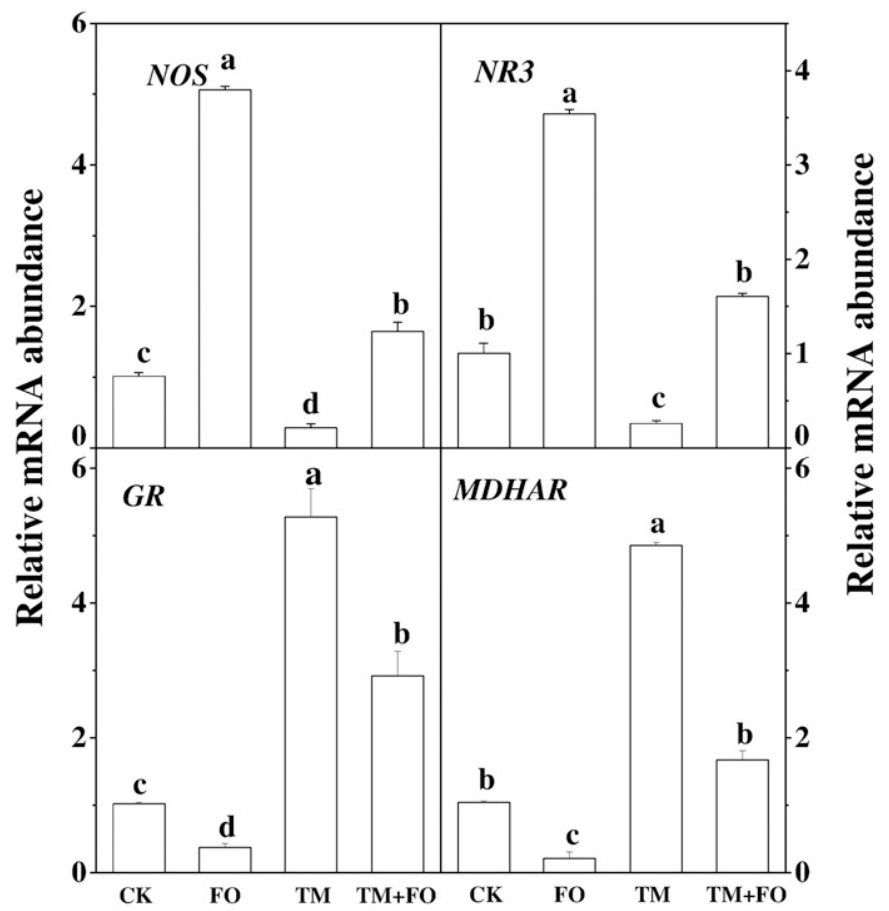

Fig. 8. Effects of Trichoderma harzianum on the expression levels of reactive oxygen species and reactive nitrogen species metabolism-related genes in response to Fusarium oxysporum in cucumber roots. Radicle tips were sampled at 1 day postinoculation with $F$. oxysporum. Data are the means of four replicates, with standard deviation shown by vertical bars. Transcript abundance was determined by quantitative reverse transcription-polymerase chain reaction and expressed as a ratio with the control, which is set as one. Means followed by different letters are significantly different at $P<0.05$ as determined by Tukey's test. CK, mock control; FO, inoculation with only $F$. oxysporum; mRNA, messenger RNA; TM, inoculation with only T. harzianum; $\mathrm{TM}+\mathrm{FO}$, coinoculation with $T$. harzianum and $F$. oxysporum. 
antioxidants cause damage and induce oxidative stress (Ahammed et al. 2019; Foyer 2018). Consistent with previous studies, F. oxysporum inoculation increased ROS accumulation in cucumber roots in this study (Figs. 3 and 4) (Chen et al. 2016). However, as noticed earlier in Indian mustard and tomato, Trichoderma inoculation remarkably decreased ROS accumulation in cucumber roots (Figs. 3 and 4) by enhancing the antioxidant potential (Ahmad et al. 2015; Bernat et al. 2018; Yu et al. 2015; Zehra et al. 2017). Consistent with our results, Mastouri et al. (2012) found that Trichoderma-colonized tomato plants showed higher activity of ROS scavenging enzymes, such as SOD, CAT, and APX, as well as AsA and GSH contents in both root and shoot, leading to enhanced tolerance to water deficit. Overexpression of Tachi (a chitinase gene from Trichoderma asperellum) in soybean (Glycine max) increases activity of POD and CAT but decreases MDA content and electrolytic leakage (percentage), thereby enhancing resistance to Sclerotinia sclerotiorum (Zhang et al. 2016b). Similarly, overexpression of a glutathione transferase (GST) gene from Trichoderma virens in tobacco alleviates oxidative stress by increasing the activities of antioxidant enzymes, such as GST, SOD, APX, POD, and CAT (Dixit et al. 2011). During colonization, T. virens secretes a group of proteins that are involved in cell wall hydrolysis, ROS scavenging, and secondary metabolism; however, the fungus also reduces POD activity in maize (Zea mays) roots as a strategy to suppress host immune responses (Nogueira-Lopez et al. 2018).

Other than ROS production, NO accumulation simultaneously occurs during the interactions between plants and pathogens (Brown et al. 2009; Cánovas et al. 2016). In the genus Arabidopsis, inoculation with $F$. oxysporum increases $\mathrm{NO}$ generation in roots (Gupta et al. 2014). Similarly, in this study, F. oxysporum inoculation caused a significant increase in NO concentration (Fig. 5). In plants, NO is synthesized mainly via two pathways. One is NR-mediated NO production, and the other is NOS-mediated NO production, although the first one is considered the most wellrecognized source of NO in plants (Lindermayr 2018; Lv et al.
2017). Therefore, we analyzed the activities of NR and NOS in cucumber roots and found that NR as well as NOS contributed to the increased NO production in response to $F$. oxysporum inoculation. However, inoculation with $T$. harzianum decreased the activities of NR and NOS, leading to decreased NO accumulation in cucumber roots accompanied with decreases of GSNO and SNO in the studied plants at 9 days after inoculation (Fig. 5). A number of reports show that NO action is dependent on its concentration and spatial production patterns (Abramowski et al. 2015; Kovacs and Lindermayr 2013). NO signal may play a positive role at the early stage. The avirulent Phytophthora infestans race rapidly enhances NO generation in potato leaves after inoculation, with the maximum $\mathrm{NO}$ accumulation at $3 \mathrm{~h}$ postinoculation (Abramowski et al. 2015). Similarly, Gupta et al. (2014) showed that $F$. oxysporum-induced rapid NO accumulation in Arabidopsis roots is closely associated with disease severity, implying that NO accumulation negatively affects plant defense. However, inoculation with T. asperelloides could effectively suppress $F$. oxysporum-induced NO production (Gupta et al. 2014). Consistent with this, we found that the genus Trichoderma could attenuate $F$. oxysporum-induced excessive NO production in cucumber roots at 9 dpi (Fig. 5). However, NO accumulation and signaling, even at the late stage, might also contribute to plant tolerance. For instance, cold acclimationinduced chilling tolerance in tomato is mediated by the increased NO accumulation until 6 days posttreatment (Lv et al. 2017), indicating the functional multiplicity of $\mathrm{NO}$ in plant stress response.

Although NO plays a critical signaling role in plant responses to stress, its very short lifetime limits the effect to the local microenvironment. However, GSNO is a relatively stable reservoir of NO and acts as a transport form of NO (Lv et al. 2017). The enzymatic recycling of GSNO is catalyzed by GSNOR. In fact, GSNOR holds a vital regulatory position at the convergence between ROS and NO signaling in plants and animals (Lindermayr 2018). In plants, GSNOR deficiency results in impaired plant disease responses (Feechan et al. 2005). Furthermore, GSNOR is

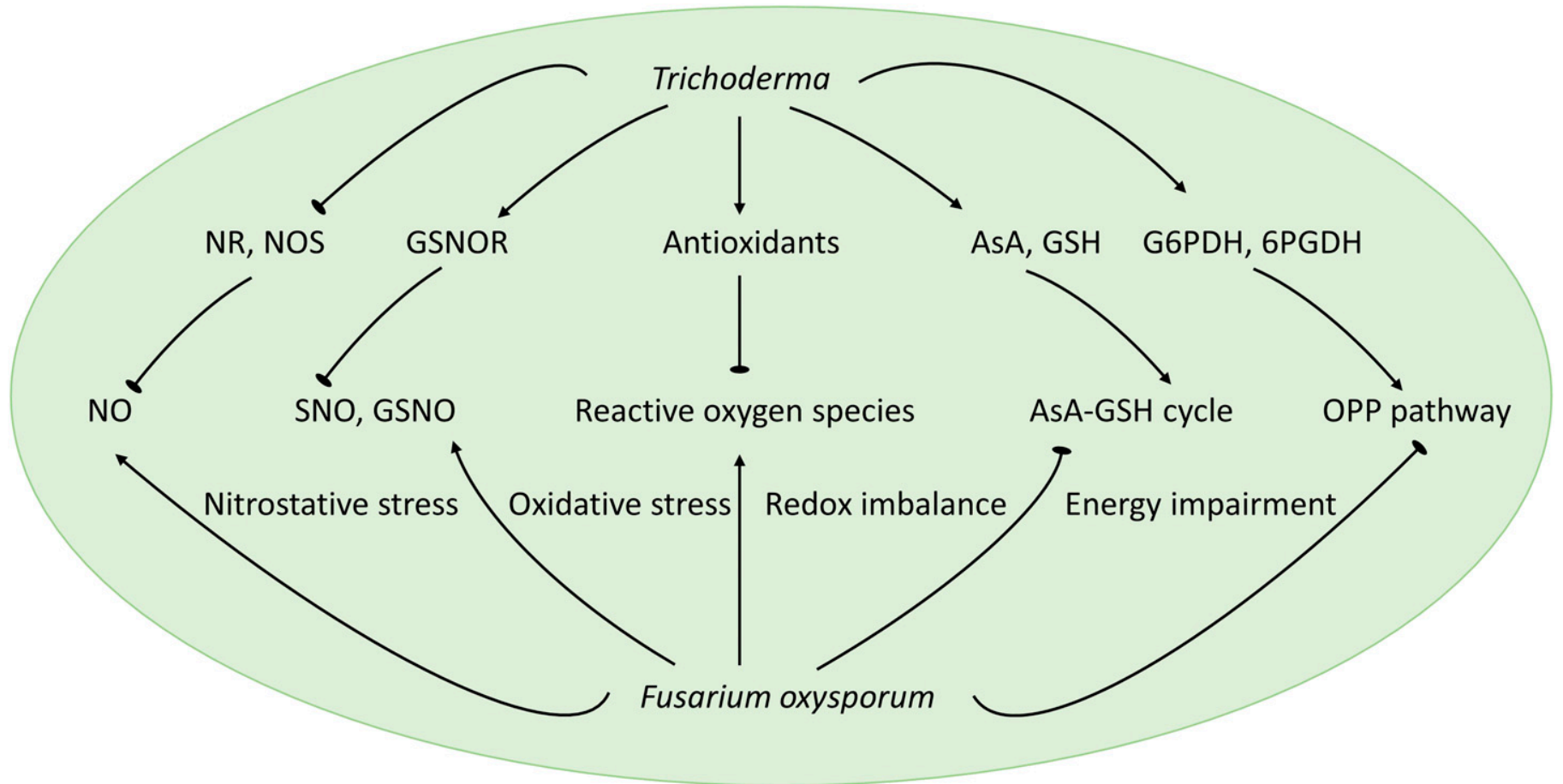

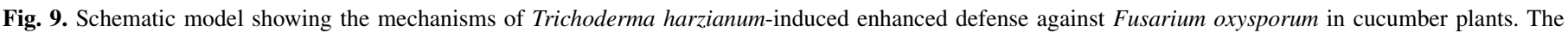

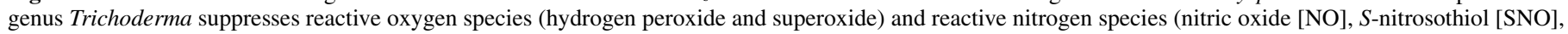

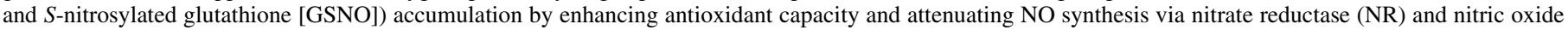

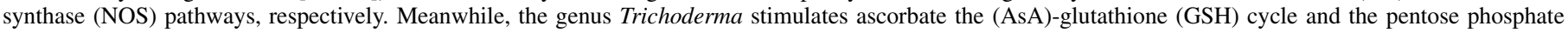

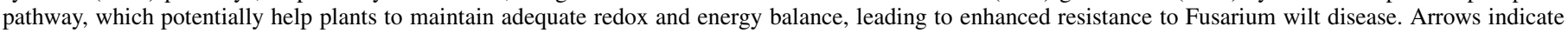

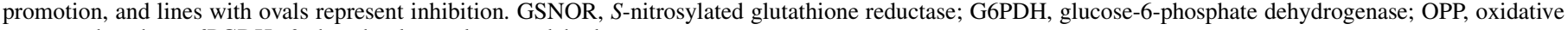
pentose phosphate; 6PGDH, 6-phosphogluconolactone dehydrogenase. 
also crucial to minimize nitrosative stress as revealed by changes in GSNO levels in plants (Chen et al. 2016; Lv et al. 2017). Here, F. oxysporum inoculation suppressed GSNOR activity but increased GSNO content, indicating that $F$. oxysporum potentially stimulated GSNO biosynthesis but did not stimulate its regeneration (Fig. 5). Similarly, F. oxysporum increased SNO content in cucumber roots, which is indirectly controlled by GSNOR activity (Lindermayr 2018). However, T. harzianum increased GSNOR activity and decreased contents of GSNO and SNO, which potentially minimized nitrostative stress.

The AsA-GSH cycle is considered the central metabolic pathway that links the $\mathrm{H}_{2} \mathrm{O}_{2}$-scavenging activity to redox signaling in plant cells. Studies revealed that the AsA-GSH cycle also plays an important role in plant-pathogen interaction (Kuźniak et al. 2017). The enhanced resistance of maize, melon, and tomato was associated with increased GSH concentration and the activity of enzymes involved in the AsA-GSH cycle (Kuźniak et al. 2017; Lanubile et al. 2012). In line with this, Trichoderma-enhanced resistance to $F$. oxysporum was attributed to increased concentrations of AsA and GSH and the activity of relevant enzymes, such as GR and MDHAR, in cucumber roots (Fig. 6 and Table 1). Furthermore, the genus Trichoderma enhanced the GaILDH and $\gamma$-GCS activities, which catalyze the rate-limiting steps of AsA and GSH biosynthesis, respectively (Chen et al. 2013). This implies that Trichoderma-induced increased AsA and GSH accumulation was attributed to their increased biosynthesis in response to Trichoderma inoculation in cucumber roots.

The OPP pathway, which is considered an important metabolic pathway in plants, has been implicated in plant responses to biotic stressors (Hu et al. 2019). Here, we showed that plant pathogenic fungus $F$. oxysporum also suppressed the OPP pathway in cucumber roots as reflected by decreased activity of relevant enzymes, such as G6PDH and 6PGDH. In nonphotosynthetic cells, such as root cells, PPP-derived reductants (NADPH) play a major role in mitigating oxidative stress by maintaining the redox potential (Kruger and von Schaewen 2003). However, Trichoderma inoculation increased the activity of the key regulatory enzymes of the OPP pathway in cucumber roots, implying that the OPP pathway plays an important role in not only the plant-Fusarium interaction toward pathogenesis but also, the Trichoderma-induced plant defense against $F$. oxysporum.

In conclusion, in this study, we showed that T. harzianum, an opportunistic filamentous fungal symbiont, could remarkably enhance plant defense against Fusarium wilt by attenuating oxidative as well as nitrostative stress in cucumber roots. Trichoderma-induced enhanced resistance to $F$. oxysporum is attributed to reduced ROS and RNS accumulation by enhanced antioxidant capacity and attenuated NO synthesis via both NR and NOS pathways, respectively. Moreover, the AsA-GSH cycle and the OPP pathway are stimulated by Trichoderma inoculation, which potentially helps plant to maintain redox balance and enhance tolerance to Fusarium wilt disease (Fig. 9). Our study shed new light on the mechanism of Trichoderma-induced wilt resistance via efficient ROS and RNS metabolism, the AsA-GSH cycle, and the OPP pathway in cucumber plants.

\section{LITERATURE CITED}

Abramowski, D., Arasimowicz-Jelonek, M., Izbiańska, K., Billert, H., and Floryszak-Wieczorek, J. 2015. Nitric oxide modulates redox-mediated defense in potato challenged with Phytophthora infestans. Eur. J. Plant Pathol. 143:237-260.

Ahammed, G. J., Li, Y., Li, X., Han, W.-Y., and Chen, S. 2018. Epigallocatechin-3-gallate alleviates salinity-retarded seed germination and oxidative stress in tomato. J. Plant Growth Regul. 37:1349-1356.

Ahammed, G. J., Xu, W., Liu, A., and Chen, S. 2019. Endogenous melatonin deficiency aggravates high temperature-induced oxidative stress in Solanum lycopersicum L. Environ. Exp. Bot. 161:303-311.

Ahmad, P., Hashem, A., Abd-Allah, E. F., Alqarawi, A. A., John, R., Egamberdieva, D., and Gucel, S. 2015. Role of Trichoderma harzianum in mitigating $\mathrm{NaCl}$ stress in Indian mustard (Brassica juncea L.) through antioxidative defense system. Front. Plant Sci. 6:868.

Airaki, M., Leterrier, M., Mateos, R. M., Valderrama, R., Chaki, M., Barroso, J. B., Del Río, L. A., Palma, J. M., and Corpas, F. J. 2012. Metabolism of reactive oxygen species and reactive nitrogen species in pepper (Capsicum апnиит L.) plants under low temperature stress. Plant Cell Environ. 35: 281-295.

Asai, S., Ohta, K., and Yoshioka, H. 2008. MAPK Signaling regulates nitric oxide and NADPH oxidase-dependent oxidative bursts in Nicotiana benthamiana. Plant Cell 20:1390-1406.

Badia, M. B., Arias, C. L., Tronconi, M. A., Maurino, V. G., Andreo, C. S., Drincovich, M. F., and Wheeler, M. C. 2015. Enhanced cytosolic NADPME2 activity in A. thaliana affects plant development, stress tolerance and specific diurnal and nocturnal cellular processes. Plant Sci. 240:193-203.

Bernat, P., Nykiel-Szymanska, J., Gajewska, E., Rozalska, S., Stolarek, P., Dackowa, J., and Slaba, M. 2018. Trichoderma harzianum diminished oxidative stress caused by 2,4- dichlorophenoxyacetic acid (2,4-D) in wheat, with insights from lipidomics. J. Plant Physiol. 229:158-163.

Bonanomi, G., Lorito, M., Vinale, F., and Woo, S. L. 2018. Organic amendments, beneficial microbes, and soil microbiota: Toward a unified framework for disease suppression. Annu. Rev. Phytopathol. 56:1-20.

Booth, C. 1971. The Genus Fusarium. Commonwealth Mycological Institute Kew, Surrey, United Kingdom.

Brown, A. J., Haynes, K., and Quinn, J. 2009. Nitrosative and oxidative stress responses in fungal pathogenicity. Curr. Opin. Microbiol. 12:384-391.

Cánovas, D., Marcos, J. F., Marcos, A. T., and Strauss, J. 2016. Nitric oxide in fungi: Is there NO light at the end of the tunnel? Curr. Genet. 62:513-518.

Chen, S., Liu, A., Zhang, S., Li, C., Chang, R., Liu, D., Ahammed, G. J., and Lin, X. 2013. Overexpression of mitochondrial uncoupling protein conferred resistance to heat stress and Botrytis cinerea infection in tomato. Plant Physiol. Biochem. 73:245-253.

Chen, S., Zhao, H., Wang, M., Li, J., Wang, Z., Wang, F., Liu, A., and Ahammed, G. J. 2017. Overexpression of E3 ubiquitin ligase gene AdbiL contributes to resistance against chilling stress and leaf mold disease in tomato. Front. Plant Sci. 8:1109.

Chen, S.-C., Zhao, H.-J., Wang, Z.-H., Zheng, C.-X., Zhao, P.-Y., Guan, Z.-H., Qin, H.-Y., Liu, A.-R., Lin, X.-M., and Ahammed, G.-J. 2016. Trichoderma harzianum-induced resistance against Fusarium oxysporum involves regulation of nuclear DNA content, cell viability and cell cycle-related genes expression in cucumber roots. Eur. J. Plant Pathol. 147:43-53.

Dixit, P., Mukherjee, P. K., Ramachandran, V., and Eapen, S. 2011. Glutathione transferase from Trichoderma virens enhances cadmium tolerance without enhancing its accumulation in transgenic Nicotiana tabacum. PLoS One 6:e16360.

Elstner, E. F., and Heupel, A. 1976. Inhibition of nitrite formation from hydroxylammoniumchloride: A simple assay for superoxide dismutase. Anal. Biochem. 70:616-620.

Estifanos Tsegaye, R., Jing, M., Jie, M., Mei, L., Beilei, W., and Xiliang, J. 2018. Biological control of soilborne pathogens (Fusarium oxysporum f. sp. cucumerinum) of cucumber (Cucumis sativus) by Trichoderma sp. J. Life Sci. 12:1-7.

Feechan, A., Kwon, E., Yun, B.-W., Wang, Y., Pallas, J. A., and Loake, G. J. 2005. A central role for $S$-nitrosothiols in plant disease resistance. Proc. Natl. Acad. Sci. USA 102:8054-8059.

Foyer, C. H. 2018. Reactive oxygen species, oxidative signaling and the regulation of photosynthesis. Environ. Exp. Bot. 154:134-142.

Foyer, C. H., and Halliwell, B. 1976. The presence of glutathione and glutathione reductase in chloroplasts: A proposed role in ascorbic acid metabolism. Planta 133:21-25.

Foyer, C. H., Valadier, M.-H., Migge, A., and Becker, T. W. 1998. Droughtinduced effects on nitrate reductase activity and mrna and on the coordination of nitrogen and carbon metabolism in maize leaves. Plant Physiol. 117:283-292.

Gordon, T. R. 2017. Fusarium oxysporum and the Fusarium wilt syndrome. Annu. Rev. Phytopathol. 55:23-39.

Gupta, K. J., Mur, L. A., and Brotman, Y. 2014. Trichoderma asperelloides suppresses nitric oxide generation elicited by Fusarium oxysporum in Arabidopsis roots. Mol. Plant-Microbe Interact. 27:307-314.

Howell, C. R. 2003. Mechanisms employed by Trichoderma species in the biological control of plant diseases: The history and evolution of current concepts. Plant Dis. 87:4-10.

$\mathrm{Hu}$, Y., You, J., Li, J., and Wang, C. 2019. Loss of cytosolic glucose-6phosphate dehydrogenase increases the susceptibility of Arabidopsis thaliana to root-knot nematode infection. Ann. Bot. 123:37-46.

Komada, H. 1975. Development of selective medium for quantitative isolation of Fusarium oxysporum from natural soil. Rev. Plant Prot. Res. 8:114-125.

Kovacs, I., and Lindermayr, C. 2013. Nitric oxide-based protein modification: Formation and site-specificity of protein $S$-nitrosylation. Front. Plant Sci. 4 : 137. 
Kruger, N. J., and von Schaewen, A. 2003. The oxidative pentose phosphate pathway: Structure and organisation. Curr. Opin. Plant Biol. 6:236-246.

Kuźniak, E., Kopczewski, T., Chojak-Koźniewska, J., 2017. Ascorbateglutathione cycle and biotic stress tolerance in plants. Pages 201-231 in: Ascorbic Acid in Plant Growth, Development, and Stress Tolerance. Springer.

Landi, M. 2017. Commentary to: "Improving the thiobarbituric acid-reactivesubstances assay for estimating lipid peroxidation in plant tissues containing anthocyanin and other interfering compounds" by Hodges et al., Planta (1999) 207:604-611. Planta 245:1067.

Lanubile, A., Bernardi, J., Marocco, A., Logrieco, A., and Paciolla, C. 2012. Differential activation of defense genes and enzymes in maize genotypes with contrasting levels of resistance to Fusarium verticillioides. Environ. Exp. Bot. 78:39-46.

Law, M. Y., Charles, S. A., and Halliwell, B. 1983. Glutathione and ascorbic acid in spinach (Spinacia oleracea) chloroplasts. The effect of hydrogen peroxide and of Paraquat. Biochem. J. 210:899-903.

Lindermayr, C. 2018. Crosstalk between reactive oxygen species and nitric oxide in plants: Key role of S-nitrosoglutathione reductase. Free Radic. Biol. Med. 122:110-115.

Liszkay, A., van der Zalm, E., and Schopfer, P. 2004. Production of reactive oxygen intermediates $\mathrm{O}_{2}^{\circ-}, \mathrm{H}_{2} \mathrm{O}_{2}$, and $\cdot \mathrm{OH}$ by maize roots and their role in wall loosening and elongation growth. Plant Physiol. 136:3114-3123.

Livak, K. J., and Schmittgen, T. D. 2001. Analysis of relative gene expression data using real-time quantitative PCR and the $2-\frac{\Delta}{T} \Delta C$ method. Methods 25: 402-408.

López-Ráez, J. A., Shirasu, K., and Foo, E. 2017. Strigolactones in plant interactions with beneficial and detrimental organisms: The Yin and Yang. Trends Plant Sci. 22:527-537.

Lorito, M., Woo, S. L., Harman, G. E., and Monte, E. 2010. Translational research on Trichoderma: From 'omics to the field. Annu. Rev. Phytopathol. 48:395-417.

Lv, X., Ge, S., Jalal Ahammed, G., Xiang, X., Guo, Z., Yu, J., and Zhou, Y. 2017. Crosstalk between nitric oxide and mpk1/2 mediates cold acclimationinduced chilling tolerance in tomato. Plant Cell Physiol. 58:1963-1975.

Ma, L. J., Geiser, D. M., Proctor, R. H., Rooney, A. P., O’Donnell, K., Trail, F., Gardiner, D. M., Manners, J. M., and Kazan, K. 2013. Fusarium pathogenomics. Annu. Rev. Microbiol. 67:399-416.

Mastouri, F., Björkman, T., and Harman, G. E. 2012. Trichoderma harzianum enhances antioxidant defense of tomato seedlings and resistance to water deficit. Mol. Plant-Microbe In. 25:1264-1271.

Mur, L. A. J., Sivakumaran, A., Mandon, J., Cristescu, S. M., Harren, F. J. M., and Hebelstrup, K. H. 2012. Haemoglobin modulates salicylate and jasmonate/ethylene-mediated resistance mechanisms against pathogens. J. Exp. Bot. 63:4375-4387.

Nogueira-Lopez, G., Greenwood, D. R., Middleditch, M., Winefield, C., Eaton, C., Steyaert, J. M., and Mendoza-Mendoza, A. 2018. The apoplastic secretome of Trichoderma virens during interaction with maize roots shows an inhibition of plant defence and scavenging oxidative stress secreted proteins. Front. Plant Sci. 9:409.

Pasqualini, S., Meier, S., Gehring, C., Madeo, L., Fornaciari, M., Romano, B., and Ederli, L. 2009. Ozone and nitric oxide induce cGMP-dependent and -independent transcription of defence genes in tobacco. New Phytol. 181:860-870.
Ramos-Martinez, J. I. 2017. The regulation of the pentose phosphate pathway. Remember Krebs. Arch. Biochem. Biophys. 614:50-52.

Rao, M. V., and Ormrod, D. P. 1995. Ozone exposure decreases UVB sensitivity in a UVB-sensitive flavonoid mutant of Arabidopsis. Photochem. Photobiol. 61:71-78.

Sakamoto, A., Ueda, M., and Morikawa, H. 2002. Arabidopsis glutathionedependent formaldehyde dehydrogenase is an $S$-nitrosoglutathione reductase. FEBS Lett. 515:20-24.

Saville, B. 1958. A scheme for the colorimetric determination of microgram amounts of thiols. Analyst (Lond.) 83:670-672.

Shan, C., and Liang, Z. 2010. Jasmonic acid regulates ascorbate and glutathione metabolism in Agropyron cristatum leaves under water stress. Plant Sci. 178:130-139.

Singh, A., Sarma, B.K., Singh, H.B., Upadhyay, R.S., 2014. Trichoderma. 533-542.

Singh, P., Tripathi, N., Joshi, D., Pathak, A. D., and Sethi, A. 2018. Trichoderma elicitors create a potential chemical barrier through induced systemic resistance against Colletotrichum falcatum and minimise sucrose losses in sugarcane. J. Plant Pathol. 100:151-162.

Tabata, K., Ôba, K., Suzuki, K., and Esaka, M. 2001. Generation and properties of ascorbic acid-deficient transgenic tobacco cells expressing antisense RNA for L-galactono-1,4-lactone dehydrogenase. Plant J. 27:139-148.

Valderrama, R., Corpas, F. J., Carreras, A., Gomez-Rodriguez, M. V., Chaki, M., Pedrajas, J. R., Fernandez-Ocana, A. N. A., Del Rio, L. A., and Barroso, J. B. 2006. The dehydrogenase-mediated recycling of NADPH is a key antioxidant system against salt-induced oxidative stress in olive plants. Plant Cell Environ. 29:1449-1459.

van der Does, H. C., and Rep, M. 2017. Adaptation to the host environment by plant-pathogenic fungi. Annu. Rev. Phytopathol. 55:427-450.

Willekens, H., Chamnongpol, S., Davey, M., Schraudner, M., Langebartels, C., Van Montagu, M., Inzé, D., and Van Camp, W. 1997. Catalase is a sink for $\mathrm{H}_{2} \mathrm{O}_{2}$ and is indispensable for stress defence in $\mathrm{C}_{3}$ plants. EMBO J. 16: 4806-4816.

Yu, Y., Yang, Z., Guo, K., Li, Z., Zhou, H., Wei, Y., Li, J., Zhang, X., Harvey, P., and Yang, H. 2015. Oxidative damage induced by heat stress could be relieved by nitric oxide in Trichoderma harzianum LTR-2. Curr. Microbiol. 70:618-622.

Zehra, A., Meena, M., Dubey, M. K., Aamir, M., and Upadhyay, R. S. 2017. Synergistic effects of plant defense elicitors and Trichoderma harzianum on enhanced induction of antioxidant defense system in tomato against Fusarium wilt disease. Bot. Stud. (Taipei, Taiwan) 58:44.

Zhang, D., Meng, K. X., Hao, Y. H., Fan, H. Y., Cui, N., Wang, S. S., and Song, T. F. 2016a. Comparative proteomic analysis of cucumber roots infected by Fusarium oxysporum f. sp. cucumerium Owen. Physiol. Mol. Plant Pathol. 96:77-84.

Zhang, F., Ruan, X., Wang, X., Liu, Z., Hu, L., and Li, C. 2016b. Overexpression of a chitinase gene from Trichoderma asperellum increases disease resistance in transgenic soybean. Appl. Biochem. Biotechnol. 180: $1542-1558$.

Zhang, J., Li, D., Wei, J., Ma, W., Kong, X., Rengel, Z., and Chen, Q. 2019. Melatonin alleviates aluminum-induced root growth inhibition by interfering with nitric oxide production in Arabidopsis. Environ. Exp. Bot. 161:157-165. 